\title{
Cultural differences in lateral biases on aesthetic judgments: The effect of native reading direction
}

\author{
Meghan E. Flath ${ }^{1}$ (D) Austen K. Smith ${ }^{1} \cdot$ Lorin J. Elias $^{1,2}$
}

Accepted: 9 March 2018/Published online: 17 March 2018

(C) The Author(s) 2018

\begin{abstract}
Left-to right (LTR) or right-to-left (RTL) directionality bias has been proposed to influence individuals' aesthetic preference for dynamic stimuli. Two general theoretical propositions attempt to account for this bias. One states that directionality bias is based on scanning habits due to cultural differences in native reading/writing direction, whereas the other proposition speculates that LTR motion bias occurs due to the right hemisphere's specialization in visuospatial processing. The current study assessed the aesthetic preference bias present when native LTR and RTL readers evaluated fashion garments on the runway in LTR or RTL motion. The aim of the study was to assess aesthetic preference bias for a novel dynamic stimulus and the corresponding influence of biological and cultural factors. Native LTR and RTL readers viewed two blocks of 20 mirror-reversed video pairs with models wearing dresses on a runway. Participants indicated which dress within the mirror-reversed pair they preferred. LTR readers displayed a significant leftward aesthetic preference bias indicating a preference for dresses moving LTR. RTL readers did not display a significant aesthetic preference bias for dresses moving in either direction. These results further support the generalizability of aesthetic preference biases for novel dynamic stimuli and support seminal literature that argues the bias occurs due to a combination of hemispheric dominance and cultural differences in native reading/writing direction.
\end{abstract}

Lorin J. Elias

lorin.elias@usask.ca

Meghan E. Flath

mef029@mail.usask.ca

1 University of Saskatchewan, Saskatoon, Canada

2 Department of Psychology, University of Saskatchewan, 9 Campus Drive, Saskatoon S7N 5A5, Canada 
Keywords Aesthetic preference bias - Directional motion · Hemispheric specialization - Laterality $\cdot$ Native reading direction

Visual stimuli, including dynamic stimuli, tend to be organized or presented in an asymmetrical manner, inducing a left-to-right aesthetic preference bias. Aesthetic preference for particular stimuli (e.g., pictures or faces) is influenced by many organizational factors such as location of mass, dimensions, implied directionality, and lighting. For example, Hutchison et al. (2011) analyzed the impact of lighting on advertisements and found that people preferred advertisements lit from above and to the left as opposed to those lit from above and to the right. Participants not only stated that they preferred the advertisement, but that they would be more likely to purchase the product (Hutchison et al. 2011). Research indicates that asymmetries within stimuli play a large role in aesthetic judgment and preference.

Two broad theoretical positions attempt to explain the underlying cause of aesthetic preference bias: native reading/writing direction and hemispheric specialization. The first theoretical stance attributes the bias to cultural differences in native reading/writing direction. This theory proposes that the direction one learns to read and write (i.e., left to right or right to left) directly impacts one's aesthetic judgment. Support for this theory has been found in the seminal aesthetic preference literature. For example, Nachson et al. (1999) assessed left-to-right and right-to-left readers' aesthetic preference for facial and body profiles. The right-to-left readers spoke either Hebrew or Arabic and the left-to-right readers spoke Russian. The researchers found that right-to-left readers preferred facial and body profiles turning to the right, whereas left-to-right readers found the opposite profiles more aesthetically appealing. Maass et al. (2007) analyzed aesthetic preference bias for stimuli in motion. Participants, whose native language was Italian, were shown mirror-reversed video clip pairs of a soccer goal and a violent film scene. For left-toright readers, a soccer goal was considered superior and more beautiful when viewed with left-to-right motion. These participants also rated the film scene as more aggressive and harmful to the victim when observed with left-to-right motion. These directionality biases were opposite in participants who read from right-to-left. The researchers concluded that scanning habits due to learned reading/writing directions influence aesthetic preference bias as opposed to hemispheric dominance.

The second theoretical stance developed to explain aesthetic preference bias states that the bias occurs due to one's innate right hemispheric dominance. Levy (1976) developed the attentional bias model and Beaumont (1985) developed the processing-efficiency model to explain the impact of hemispheric specialization on aesthetic preference. According to Levy (1976), visuospatial processing induces activation of the right hemisphere, in turn, promoting attentional bias to the left visual field. Therefore, people prefer images with a larger portion of the mass on the right-hand side because it causes the leftward attentional bias to be negated (Levy 1976). Beaumont (1985) agreed with this notion but added to Levy's (1976) theory when he discovered that his participants were displaying a right gaze bias. Beaumont (1985) reasoned that when people attend to objects in the left visual field, the information travels contralaterally to the right hemisphere. According to his processing-efficiency model, this right gaze bias allows more of the visual scene to 
fall into the left visual field and be processed by the right hemisphere (Beaumont 1985). This creates an efficient alignment between the stimulus and proper circuitry due to the right hemisphere's specialization for visuospatial processing.

Numerous studies have supported this hemispheric specialization theory. Mead and McLaughlin (1992) discovered that dextral and inverted sinistral participants found pictures with an implied left-to-right directionality more aesthetic pleasing as compared to their mirror-reversed pairs. Left-to-right motion will promote a rightward gaze, causing a majority of the picture to be efficiently processed by the right hemisphere, further supporting Beaumont's (1985) processing-efficiency model. Furthermore, Treiman and Allaith (2013) analyzed the impact of reading direction on aesthetic preference bias for pictures. They assessed participants who either read Arabic or English and found that those who read right-to-left displayed no significant difference in preference bias as compared to left-to-right readers. Both groups exhibited an aesthetic preference bias for right-facing pictures, bringing into question the impact of native reading/writing direction and further supporting the hemispheric specialization theory.

Certain research claims the combination of cultural differences in native reading/ writing direction and hemispheric dominance to be the underlying cause of aesthetic preference bias. Andrews (2014) found that native reading/writing direction influenced lighting bias, but also discovered that hemispheric dominance plays a role. According to her research, the degree of lighting bias decreases as people age due to the deterioration of the right hemisphere. The researcher also discovered that right hemispheric lesions caused a contralesional shift in lighting bias. Friedrich et al. (2014) aimed to assess the prevalence of aesthetic preference bias when analyzing landscapes and mobile objects. They analyzed both right-to-left and leftto-right readers to determine if the aesthetic preference bias was due to native reading/writing direction or hemispheric specialization. The researchers found that left-to-right readers displayed a left-to-right directionality bias and mass location had no corresponding impact. Right-to-left readers did not display a bias. The directionality bias was stronger for the video stimuli in both groups of readers. The researchers concluded that hemispheric specialization is the basis for aesthetic preference bias and these learned scanning habits due to reading direction modulate the strength of the bias.

Research has also found that this aesthetic preference bias is further modulated by gender. Friedrich et al. (2014) discovered that their male participants displayed a stronger left-to-right directionality bias than females. This may be due to differential lateralization between males and females (Amunts et al. 2007; Cela-Conde et al. 2009). Males display a more dominant right hemispheric lateralization in visuospatial processing (Amunts et al. 2007; Friedrich et al. 2014). Furthermore, when evaluating stimuli for aesthetic appeal, males have been shown to solely display activation in the right hemisphere, whereas females express bilateral activation (Cela-Conde et al. 2009). This differential effect of gender has also been observed in line bisection tasks where males exhibit a stronger leftward bias than females (Jewell and McCourt 2000; Roig and Cicero 1994). The combination of these findings may account for males enhanced left-to-right directionality bias. 
Visual scanning habits and eye movements may also contribute to aesthetic preference bias. Previous literature has stated that both one's native reading/writing direction and hemispheric lateralization influence scanning and eye movements. The leftward bias observed in facial processing research has been attributed to an interaction between scanning patterns and hemispheric specialization (Megreya and Havard 2011; Vaid and Singh 1989). Vaid and Singh (1989) aimed to assess the underlying processes involved in the leftward bias found in facial affect processing. The researchers looked at both left-to-right (Hindi) and right-to-left (Arabic or Urdu) readers and found that the leftward bias was significantly stronger in the leftto-right readers. The right-to-left readers did not display a significant bias. They concluded that the right-to-left scanning patterns exhibited by the right-to-left readers negated the leftward bias due to hemispheric specialization. Smith and Elias (2013) went on to study the impact of reading direction and eye movements on lighting biases. They found that left-to-right readers displayed more fixations on the left side of the image, whereas right-to-left readers exhibited the opposite. Left-toright readers revealed significant preference for images lit from the left and, although not significant, right-to-left readers showed a preference for images lit from the right. Abed (1991) analyzed the impact of reading direction on fixation location and saccade direction. He found no different in fixation location; however, left-to-right readers displayed more left-to-right saccadic movements, whereas right-to-left readers displayed the opposite. These studies indicate that eye movements are further influencing aesthetic preference bias.

The purpose of the current study is to assess aesthetic preference biases for a novel dynamic stimulus and to explore the impact of cultural factors, such as native reading/writing direction, and biological factors on these preference biases. The current study will evaluate the aesthetic preference bias for a garment in motion and the corresponding eye movements for native left-to-right and right-to-left readers. We predict that left-to-right readers will prefer garments moving left to right, whereas right-to-left readers will display no aesthetic preference bias due to a combined effect of hemispheric dominance and native reading direction. We hypothesize that males will display an overall stronger leftward bias than females. Furthermore, we predict that left-to-right and right-to-left readers will display a shorter fixation duration average for dynamic stimuli in line with their respective reading directions. Finally, we predict that left-to-right and right-to-left readers will exhibit faster saccadic velocity averages when exploring dynamic stimuli moving in the same direction as their native reading direction.

\section{Method}

\section{Participants}

Seventy-eight participants with a mean age of 25.9 years $(S D=6.356$, range 17-39 years) voluntarily participated in this study. There were 50 participants (36 females) whose native reading direction was left to right, and 28 participants (17 females) whose native reading direction was right to left. Right-to-left readers' 
native language was Arabic, Urdu, or Farsi and the length of time right-to-left readers had been fluent in a left-to-right language ranged from 7 months to 26 years. Participant recruitment occurred through the use of the University of Saskatchewan Psychology Participant Pool, a recruitment poster, and a recruitment email list collected from previous studies. Participants recruited through the Participant Pool received one bonus mark toward their 100 or 200-level psychology course. Those recruited from the email list received 10 dollars in compensation for their participation. Participants were recruited after the study received ethical approval from the University of Saskatchewan Behavioural Research Ethics Board on June 16, 2016 (Beh 16-191). Participant Pool participants who did not meet the study inclusion criteria could still participate for credit; however, their data would not be used in the analysis and therefore would not contribute to the results.

\section{Stimuli and procedure}

Each participant was exposed to three video pair practice trials followed by two blocks of 20 mirror-reversed video stimuli for a total of 80 video clips. The video clip pairs were a forced-choice task and contained a female model wearing a dress and walking down the runway from left to right or right to left. Each video pair contained a different dress than the previous pair. The participants viewed the dress moving down the runway from left to right and right to left and chose which version of the dress they preferred. Having the participants choose between the same dress moving in different directions ensured that the participants' preference choice was due to directionality and not influenced by confounding factors such as lighting or dress colour. No deception occurred, and the participants were not led to believe that the two dresses in the video pairs were different.

These video clips were taken from two Ellie Saab runway shows found on YouTube and cut into two-second clips using iMovie. Lighting came from the same direction in both fashion shows and was counterbalanced by mirror-reversing each video. These two-second video clips were displayed on a computer screen using SMI Experiment Suite 360. Two versions of the experimental stimuli were created to counterbalance the direction of motion in the video pairings. Half the participants completed version one, where a random selection of video pairings was presented with the leftward motion first and the remaining pairings had the rightward motion presented first. The other half of the participants completed version two, which was exactly opposite to version one.

Participants arrived at the lab and informed consent was obtained prior to beginning the experiment. Each participant completed a demographics questionnaire along with a handedness and footedness questionnaire (Elias and Bryden 1998). This ensured that handedness and footedness were accounted for as covariates. Participants were randomly assigned to complete one of the two versions of the experiment and were instructed to sit down in front of a computer screen and place their chin on the chin rest in order for the eye tracker to track their eye movements. They then completed an eye tracker calibration. Once calibrated, instructions were verbally presented to them from a script. After the instructions, the researcher hit the space bar to begin the experiment. A fixation cross appeared at the 
centre of the screen for $1000 \mathrm{~ms}$ before each pair of videos. The pair of videos then played, and a question appeared asking participants to verbally indicate whether they preferred the first or second dress. The researcher recorded their responses on a separate computer. While participants were completing the experimental task, A SensoMotoric Instruments (SMI) Remote Eye Tracking Device (RED 4) recorded their eye movements with a frequency of $60 \mathrm{~Hz}$. The eye data was transferred to iView $\times$ operating on an SMI custom built PC with a $2.4 \mathrm{GHz}$ Intel CPU and a $580 \mathrm{MHz}$ Nvidia GeForce 9300 M GS graphics card. Experiment Center was used to present the stimuli on a $1280 \times 1024$ resolution computer monitor. The distance from the head to the screen was $70 \mathrm{~cm}$. Once the experiment was complete, participants were asked if they had any questions and were handed a debriefing form. The eye-tracking data was analyzed through a specific program called Begaze.

\section{Scoring}

Participants were assigned a -1 every time they chose a leftward moving garment and $a+1$ every time they chose a rightward moving garment. The participant's scores for each video pair were averaged to assign them an overall mean aesthetic bias score.

\section{Results}

\section{Aesthetic preference scores}

The dependent variable was the participant's mean aesthetic bias score and a 2 (Reading direction [LTR, RTL]) $\times 2$ (Gender [male, female]) Independentmeasures ANOVA was conducted. The overall bias scores were determined by adding the number of right-to-left and left-to-right selections. There was a significant main effect of reading direction where the mean aesthetic bias scores of left-to-right and right-to-left readers significantly differed from one another, $F(1$, $77)=10.472, p=.002$. Left-to-right readers displayed a leftward aesthetic preference bias $(-.2014)$, and right-to-left readers displayed a slight rightward bias (.0837). No significant main effect of gender was found and no significant interaction between gender and native reading direction was found (see Fig. 1).

One-sample $t$ tests were then conducted to determine whether a significant aesthetic bias was present in both groups of readers. Left-to-right readers exhibited a significant left-to-right aesthetic bias, $t(49)=-4.118, p<.001$. However, the mean aesthetic bias score for right-to-left readers did not significantly differ from zero, $t(27)=1.075, p=.292$.

\section{Eye-tracking scores}

The dependent variable is the amount of time (ms) each participant spent fixating on the dynamic stimuli and a 2 (Reading direction [LTR, RTL]) $\times 2$ (Fixation duration average [LTR videos, RTL videos]) mixed-measures ANOVA was conducted to 


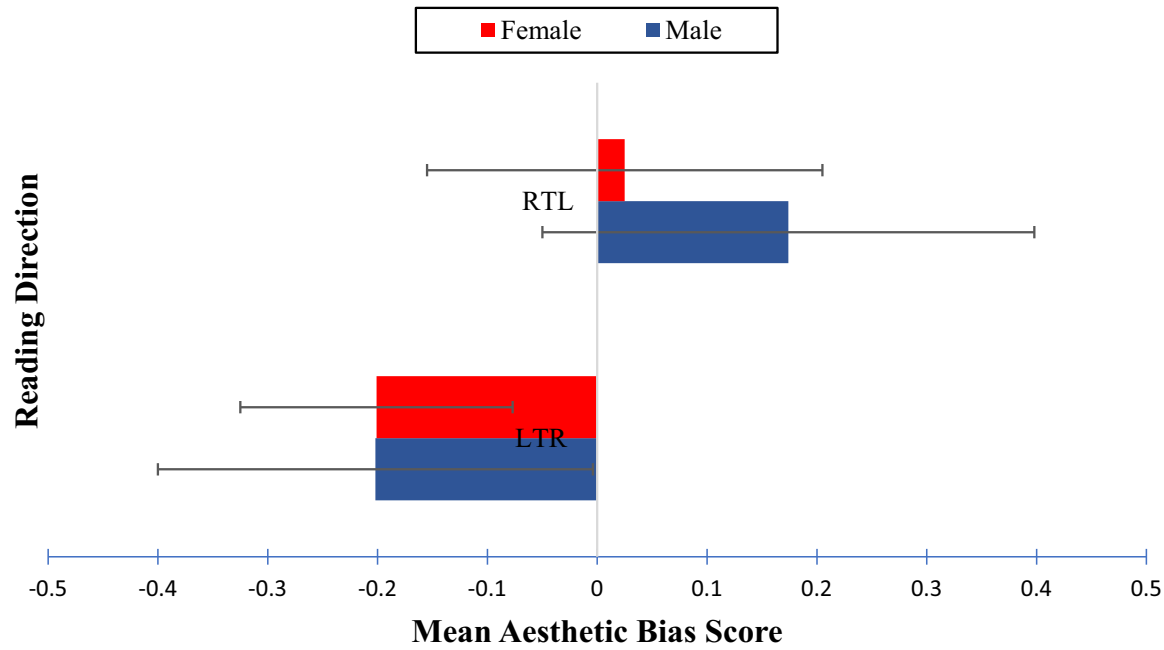

Fig. 1 The aesthetic preference bias scores for male and female right-to-left (RTL) and left-to-right (LTR) readers. The error bars attached to each column represent a $95 \%$ confidence interval

analyze fixation duration for the dynamic stimuli. No significant main effects or interaction were found. A 2 (Reading direction [LTR, RTL]) $\times 2$ (Saccadic velocity average [LTR videos, RTL videos]) mixed-measures ANOVA was conducted to analyze whether the speed of saccadic movements differed depending on the combination or reading direction and video direction. The dependent variable is the velocity $(\%)$ of the saccadic movements. No significant main effects or interaction were found. The significance level was set to $\alpha=0.05$ for all statistical tests.

\section{Discussion}

The main purpose of this study was to analyze the impact of native reading/writing direction on aesthetic preference biases for a novel dynamic stimulus and to clarify whether hemispheric specialization and/or native reading/writing direction modulated the bias. The results supported the first hypothesis, indicating that left-to-right readers exhibited a left-to-right preference bias and right-to-left readers displayed no bias. Furthermore, these results further support seminal literature which argues that aesthetic preference biases are mediated by an interaction between hemispheric dominance and native reading/writing direction (Andrews 2014; Chokron and De Agostini 2000; Friedrich et al. 2014; Maass and Russo 2003; Nicholls and Roberts 2002). In keeping with Friedrich et al.'s (2014) study, these results support Beaumont's (1985) processing-efficiency model. The left-to-right directionality of the dynamic stimuli pulled the participants' gaze to the right side of the visual scene. This caused a larger portion of the scene to fall into the left visual field, facilitating efficient processing by the right hemisphere and producing a preference for left-to-right directionality. Learning to read and write from right-to-left may 
have produced scanning habits or past cultural experiences that balanced the right hemisphere efficiency effect, creating no directionality bias for this reading group (Friedrich et al. 2014; Smith and Elias 2013).

No significant differences were found for the eye-tracking data. There were no significant differences between reading direction and video direction for fixation duration average or saccadic velocity average. Our results do not correspond with the seminal research on the impact of native reading/writing direction on aesthetic preference biases. The seminal research suggests that reading direction impacts aesthetic preference biases through learned eye-tracking patterns (Abed 1991; Megreya and Havard 2011; Smith and Elias 2013; Vaid and Singh 1989). In the seminal literature participants were free-viewing static images, whereas in the current study they had a fixed amount of time to view the dynamic stimuli. The dynamic nature of the stimuli in the current study may have influenced the manner in which the participants visually scanned the video clips. Participants' eyes may have simply followed the motion of the dynamic stimuli.

There are multiple potential reasons why the eye-tracking results of the current study do not align with seminal literature. First, learned scanning habits should not be ruled out as one of the underlying causes for aesthetic preference biases. The program utilized for the eye-tracking data analysis did not allow us to assess other measures such as the direction of saccadic movements or the location of fixations. Further eye-tracking data analysis may be required to find the underlying differences in the way right-to-left and left-to-right readers explore the dynamic stimuli. Second, eye-tracking is a measure of overt, not covert, visual attention. Participants may be overtly attending to the dynamic stimuli in the same manner, but covertly visually studying the stimuli in a different manner. The eye-tracking data analysis would not be able to assess this potential covert visual attention difference that may be driving the aesthetic preference biases in left-to-right and right-to-left readers. Finally, the two groups of readers may be visually exploring the dynamic stimuli in the same way but making different choices due to past experiences and familiarity. Dynamic stimuli provide participants with a target to follow, potentially causing the two groups of readers to visually attend to the moving stimuli in the same way. The different preference choices made by the two groups of readers may be partially due to past cultural experiences, regardless of the way they are visually attending to the stimuli. One's preference for directional motion may be influenced by what appears natural to them and in line with their native reading/writing direction.

Lastly, the hypothesis regarding the differential effects of gender was not found to be significant. Aesthetic preference bias did not significantly differ between males and females. This is inconsistent with previous literature that states that males tend to display a stronger leftward aesthetic preference bias than females due to their increased right hemispheric lateralization in visuospatial processing (Amunts et al. 2007; Friedrich et al. 2014). One reason may be due to the disproportionate sample size. This study tested a total of 53 females and only 25 males. Furthermore, males may have been unfamiliar with the stimuli or attended less to the stimuli than females. Future research needs to be done to determine the underlying reason for 
these inconsistent results and whether this gender difference resurfaces with other dynamic stimuli.

The results of this study further support the presence of aesthetic preference bias and its generalization to a novel dynamic stimulus. Furthermore, the findings that left-to-right readers display a significant leftward bias and right-to-left readers display no bias are in accordance with the results and conclusions found in previous literature (Chokron and De Agostini 2000; Friedrich et al. 2014). This seminal literature argues that aesthetic preference biases are mediated by a combination of hemispheric dominance and native reading/writing direction. All people may have an underlying hemispheric specialization that promotes leftward aesthetic preference biases due to processing efficiency; however, learned scanning directions or past cultural experiences may have the ability to eliminate this leftward bias.

This study is not free of limitations. The main limitation is that a majority of the right-to-left readers were bilingual, and the forms, instructions, and questionnaires were presented in English. This could have weakened the degree of bias displayed by right-to-left readers, however a significant difference in aesthetic preference bias was still found between both reading direction groups. In future research, experimental protocol could include forms in participants' native language and ensure participants are primed in their native language prior to beginning the experiment.

In conclusion, one's native reading/writing direction appears to have a significant impact on aesthetic preference bias for dynamic stimuli with an underlying and combined impact of hemispheric specialization. This research assesses a novel dynamic stimulus, demonstrating the generalizability of aesthetic preference biases and contributing to the comparison of these biases for dynamic and static stimuli. Future research should assess the strength of aesthetic biases present in fully monolingual readers, further analyze eye tracking data, and determine the generalizability of this aesthetic bias to other dynamic stimuli.

Acknowledgements Natural Sciences and Engineering Research Council of Canada.

\section{Compliance with ethical standards}

Conflict of interest The authors declare that they have no conflict of interest.

Open Access This article is distributed under the terms of the Creative Commons Attribution 4.0 International License (http://creativecommons.org/licenses/by/4.0/), which permits unrestricted use, distribution, and reproduction in any medium, provided you give appropriate credit to the original author(s) and the source, provide a link to the Creative Commons license, and indicate if changes were made.

\section{References}

Abed, F. (1991). Cultural influences on visual scanning patterns. Journal of Cross-Cultural Psychology, 22(4), 525-534. https://doi.org/10.1177/0022022191224006. 
Amunts, K., Armstrong, E., Malikovic, A., Hömke, L., Mohlberg, H., Schleicher, A., et al. (2007). Gender-specific left-right asymmetries in human visual cortex. The Journal of neuroscience, 27(6), 1356-1364. https://doi.org/10.1523/JNEUROSCI.4753-06.2007.

Andrews, B. (2014). The assumed light source direction: Evidence from different populations. Doctoral dissertation, Prifysgol Bangor University.

Beaumont, J. G. (1985). Lateral organization and aesthetic preference: The importance of peripheral visual asymmetries. Neuropsychologia, 23(1), 103-113. https://doi.org/10.1523/JNEUROSCI.475306.2007.

Cela-Conde, C. J., Ayala, F. J., Munar, E., Maestú, F., Nadal, M., Capó, M. A., et al. (2009). Sex-related similarities and differences in the neural correlates of beauty. Proceedings of the National Academy of Sciences, 106(10), 3847-3852. https://doi.org/10.1073/pnas.0900304106.

Chokron, S., \& De Agostini, M. (2000). Reading habits influence aesthetic preference. Cognitive Brain Research, 10(1), 45-49. https://doi.org/10.1016/S0926-6410(00)00021-5.

Elias, L. J., \& Bryden, M. P. (1998). Footedness is a better predictor of language lateralisation than handedness. Laterality: Asymmetries of Body, Brain and Cognition, 3(1), 41-52. https://doi.org/10. 1080/713754287.

Friedrich, T. E., Harms, V. L., \& Elias, L. J. (2014). Dynamic stimuli: Accentuating aesthetic preference biases. Laterality: Asymmetries of Body, Brain and Cognition, 19(5), 549-559. https://doi.org/10. 1080/1357650X.2014.886585.

Hutchison, J., Thomas, N. A., \& Elias, L. (2011). Leftward lighting in advertisements increases advertisement ratings and purchase intention. Laterality: Asymmetries of Body, Brain and Cognition, 16(4), 423-432. https://doi.org/10.1080/13576501003702663.

Jewell, G., \& McCourt, M. E. (2000). Pseudoneglect: A review and meta-analysis of performance factors in line bisection tasks. Neuropsychologia, 38(1), 93-110. https://doi.org/10.1016/S00283932(99)00045-7.

Levy, J. (1976). Lateral dominance and aesthetic preference. Neuropsychologia, 14(4), 431-445. https:// doi.org/10.1016/0028-3932(76)90072-5.

Maass, A., Pagani, D., \& Berta, E. (2007). How beautiful is the goal and how violent is the fistfight? Spatial bias in the interpretation of human behavior. Social Cognition, 25(6), 833-852. https://doi. org/10.1521/soco.2007.25.6.833.

Maass, A., \& Russo, A. (2003). Directional bias in the mental representation of spatial events nature or culture? Psychological Science, 14(4), 296-301. https://doi.org/10.1111/1467-9280.14421.

Mead, A. M., \& McLaughlin, J. P. (1992). The roles of handedness and stimulus asymmetry in aesthetic preference. Brain and Cognition, 20(2), 300-307. https://doi.org/10.1016/0278-2626(92)90022-E.

Megreya, A. M., \& Havard, C. (2011). Left face matching bias: Right hemisphere dominance or scanning habits? Laterality, 16(1), 75-92. https://doi.org/10.1080/13576500903213755.

Nachson, I., Argaman, E., \& Luria, A. (1999). Effects of directional habits and handedness on aesthetic preference for left and right profiles. Journal of Cross-Cultural Psychology, 30(1), 106-114. https:// doi.org/10.1177/0022022199030001006.

Nicholls, M. E., \& Roberts, G. R. (2002). Can free-viewing perceptual asymmetries be explained by scanning, pre-motor or attentional biases? Cortex, 38(2), 113-136. https://doi.org/10.1016/S00109452(08)70645-2.

Roig, M., \& Cicero, F. (1994). Hemisphericity style, sex, and performance on a line-bisection task: An exploratory study. Perceptual and Motor Skills, 78(1), 115-120. https://doi.org/10.2466/pms.1994. 78.1 .115 .

Smith, A. K., \& Elias, L. J. (2013). Native reading direction and corresponding preferences for left-or right-lit images. Perceptual and Motor Skills, 116(2), 355-367. https://doi.org/10.2466/23.24.PMS. 116.2.355-367.

Treiman, R., \& Allaith, Z. (2013). Do reading habits influence aesthetic preferences? Reading and Writing, 26(8), 1381-1386. https://doi.org/10.1007/s11145-012-9424-1.

Vaid, J., \& Singh, M. (1989). Asymmetries in the perception of facial affect: Is there an influence of reading habits? Neuropsychologia, 27(10), 1277-1287. https://doi.org/10.1016/00283932(89)90040-7. 